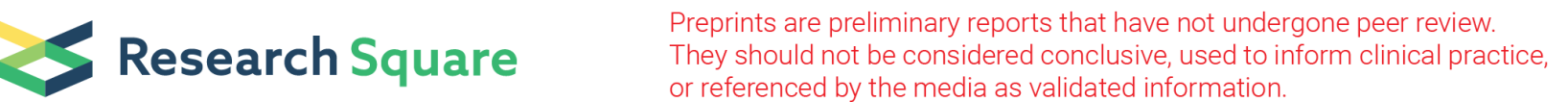 \\ Dexmedetomidine reduces isoflurane-induced neuroapoptosis through regulating BDNF and proBDNF
}

\section{Xudong Ding}

China Medical University Shengjing Hospital Nanhu Branch: Shengjing Hospital of China Medical University

\section{Hui Zhang}

China Medical University Shengjing Hospital Nanhu Branch: Shengjing Hospital of China Medical University

\section{Shiwei Sun}

China Medical University Shengjing Hospital Nanhu Branch: Shengjing Hospital of China Medical University

\section{Chenguang Ma}

China Medical University Shengjing Hospital Nanhu Branch: Shengjing Hospital of China Medical University

\section{NINGNING ZHENG ( $\nabla$ nnzheng@cmu.edu.cn )}

China Medical University https://orcid.org/0000-0002-9728-2651

\section{Hongtao Liu}

China Medical University Shengjing Hospital Nanhu Branch: Shengjing Hospital of China Medical University

\section{Research}

Keywords: Dexmedetomidine, Neuroapoptosis, Isoflurane, a2-adrenergic agonist, Neurotrophin, BDNF, proBDNF, ERK, JNK

Posted Date: June 9th, 2021

DOl: https://doi.org/10.21203/rs.3.rs-596903/v1

License: (c) (1) This work is licensed under a Creative Commons Attribution 4.0 International License. Read Full License 


\section{Abstract}

Background: It is well-acknowledged that Isoflurane induces neuroapoptosis in neonatal rats. Dexmedetomidine, as an a2-adrenergic agonist, was previously demonstrated to provide neuroprotection when administered during isoflurane anesthesia. Our study aims to investigate the mechanisms concerning the neuroprotective effect of dexmedetomidine from the alterations of BDNF,ERK, and JNK signals in the hippocampal region.

Methods: Neonatal Sprague-Dawley rats at postnatal day 7 were assigned into Control group, Isoflurane group, Dexmedetomidine group and Inhibitor group. After exposed to $2 \%$ isoflurane in $40 \%$ of oxygen mixed with nitrogen for $4 \mathrm{~h}$, the hippocampus tissues were separated and critical signal pathway proteins of BDNF, proBDNF, JNK, ERK, and caspase 3 were detected.

Results: Neuroapoptosis was triggered by Isoflurane with the increased expression of caspase 3 and TUNEL-positive cells. This effect was reversed by dexmedetomidine accompanying with up-regulation of BDNF and phospho-ERK and down-regulation of proBDNF and phospho-JNK.

Conclusions: This study revealed that dexmedetomidine pretreatment can attenuate neurotoxicity caused by isoflurane in neonatal rats by regulating BDNF, proBDcNF, ERK, and JNK, which would provide a new target for neuroprotection.

\section{Background}

With the rapid development of medical technology, growing numbers of surgical procedures can be carried out in infants under general anesthesia. It is reported that some non-obstetric surgery of pregnant women under general anesthesia resulted in later learning and memory deficits in the immature fetal brain of infants. Inhaled anesthetics have a significant advantage in general anesthesia in infants due to their characteristics of high anesthetic efficacy and strong maneuverability. Without the respiratory irritation, isoflurane is widely used in clinical anesthesia in infants due to the rapid induction and awakening properties and easy adjustment of anesthesia depth. In recent years, a multitude of experiments focus on the effects of isoflurane on the cognitive function, and some of the studies have found that prolonged exposure to isoflurane in children may cause acute cognitive dysfunction. It is well known that anesthetics may cause apoptotic neurodegeneration in the neonatal brain in rats, and results in subsequent cognitive dysfunction in adulthood [1] [2][3][4] [5] [6]. A crucial factor mediating anesthetic neurotoxicity is that the brain is more vulnerable to injury during the period of synaptogenesis than any other phase [7]. It is reported that isoflurane neurotoxicity is partially mediated by the action of proBDNF/p75NTR (p75 neurotrophin receptor), and the inhibitor of p75NTR was able to attenuate this neurotoxicity [8][9].

Dexmedetomidine is a highly selective agonist of a2-adrenergic receptors which is widely used in clinical practice with a broad spectrum of effects, including clinical sedation, anesthetic sparing effects, and analgesia [10] [11]. In addition, dexmedetomidine has gained widespread attention for its neuroprotective 
properties on different experimental models of brain injury [12] [13] [14] [15] [16] [17] [18]. Recent experimental works indicate that dexmedetomidine reduced the isoflurane-induced neurotoxicity in neonatal rats [13], but the mechanism remains unclear. Several studies found that dexmedetomidine could improve cognitive function after the operation. Using in vitro approaches, it has been suggested that the neuroprotective mechanism of dexmedetomidine may be involved in the regulation of brainderived neurotrophic factor (BDNF) expression [19]. BDNF is the product of proneurotrophin (proBDNF). BDNF activation of tropomyosin receptor kinase $B(T r k B)$ enhances neurite growth and causes cell differentiation, and signaling by proBDNF through p75NTR induces cell apoptosis [9]. Activation of TrkB leads to extracellular signal-regulated kinase (ERK) phosphorylation, which is essential to cell survival. In contrast, the activation of proBDNF/p75NTR induces c-Jun N-terminal kinase (JNK) phosphorylation, which is critical for cellular apoptosis.

Despite the proposed mechanisms concerning the neuroprotective effect of dexmedetomidine, the possible interaction between dexmedetomidine and proBDNF has not been explored. Our experiment was designed to investigate whether dexmedetomidine pretreatment can attenuate neurotoxicity caused by isoflurane in neonatal rats by regulating the expressions of BDNF, proBDNF, phospho-ERK, and phosphoJNK in the hippocampal region.

\section{Methods}

\section{Animal and group}

Seven-day-old (P7) Sprague-Dawley rat pups (Liaoning Changsheng Biotechnology Co. Ltd., permission number: SCXK 2015-0001) weighing (15.2囚3.2)g were used because this is the time when they are vulnerable to anesthesia-induced neurologic deficits. Seventy-two neonatal Sprague-Dawley rats at postnatal day 7 were assigned randomly to four groups: Control group (group Con) where rats were injected $150 \mu \mathrm{l}$ saline intraperitoneally; Isoflurane group (group Iso) where rats were injected intraperitoneally with $150 \mu$ l saline; Dexmedetomidine group (group Dex) where rats were injected intraperitoneally with dexmedetomidine $50 \mu \mathrm{g} / \mathrm{kg}$ in $150 \mu \mathrm{l}$ saline; Inhibitor group (group Inh) where rats were injected intraperitoneally with (dexmedetomidine 50 $\mu \mathrm{g} / \mathrm{kg}+$ TAT-PEP5 $10 \mu \mathrm{M} / \mathrm{kg}+\mathrm{K} 252 \mathrm{a} 80 \mu \mathrm{g} / \mathrm{kg}$ ) in $150 \mu$ saline.

\section{Anesthetic Neurotoxicity Model}

Seven-day-old Sprague-Dawley rat pups were exposed $4 \mathrm{~h}$ to $2.0 \%$ isoflurane in $40 \%$ oxygen in a chamber $(\mathrm{n}=18$ per group). Saline or dexmedetomidine $(50 \mu \mathrm{g} / \mathrm{kg})$ or TrkB inhibitor K252a $(80 \mu \mathrm{g} / \mathrm{kg})$ or p75NTR inhibitor TAT-PEP5 $(10 \mu \mathrm{M} / \mathrm{kg})$ were injected intraperitoneally 15 minutes before the exposure. Rats in group Iso received $2 \%$ isoflurane and saline $(150 \mu \mathrm{l})$; rats in group Dex received $2 \%$ isoflurane and dexmedetomidine $(50 \mu \mathrm{g} / \mathrm{kg}$ intraperitoneally); rats in group Inh received $2 \%$ isoflurane and dexmedetomidine $(50 \mu \mathrm{g} / \mathrm{kg})$ and $\mathrm{K} 252 \mathrm{a}(80 \mu \mathrm{g} / \mathrm{kg}, \mathrm{CST})$ and TAT-PEP5 $(10 \mu \mathrm{M} / \mathrm{kg}$, millipore). Rats in group Con received $40 \%$ oxygen and saline $(150 \mu$ intraperitoneally). 
Some animals were sacrificed (with sodium pentobarbital $100 \mathrm{mg} / \mathrm{kg}$ intraperitoneally) 2 hours after the end of gas exposure. Their hippocampi were isolated immediately on ice and then stored at $-80^{\circ} \mathrm{C}$ until used for western blot study $(n=8)$. The other rats were sacrificed 2 hours after the end of gas exposure and perfused transcardially with ice-cold saline followed by $4 \%$ paraformaldehyde in $0.1 \mathrm{M}$ phosphate buffer. Their brains were post-fixed for $48 \mathrm{~h}$, and then dehydrated and embedded in paraffin. The paraffin blocks stored at $4{ }^{\circ} \mathrm{C}$, and then used for TUNEL and immunohistochemistry study $(n=10)$.

\section{TUNEL fluorescent assay}

The paraffin blocks were separated from tissue holders, $3.5 \mu \mathrm{m}$ sections were then cut, placed on glass slides. The paraffin sections were allowed to dry at $67^{\circ} \mathrm{C}$ for $24 \mathrm{~h}$, and then removed paraffin and rinsed in phosphate-buffered saline (PBS). TUNEL fluorescent assay was performed using the In Situ Cell Death Detection Kit, POD (Roche, USA) according to the manufacturer's protocol. Tissue sections were incubated by proteinase $\mathrm{K}$ solution for 20 minutes, exposed to equilibration buffer for 10 minutes and the terminal deoxynucleotidyl transferase (TdT) and incubated in a humidified chamber at $37^{\circ} \mathrm{C}$ for 1 hour, and the slides were protected from direct light during the experiment. Afterward, the reaction was stopped, and then the nuclei staining with DAPI. For negative-controls, the slides were exposed to reaction buffer without TdT. Images were acquired at $400 x$ by NIS-Elements F 3.0 software linked to Nikon E800 microscope. TUNEL positive cells in the hippocampus were analyzed with Image-Pro Plus software. The apoptotic index was defined as the percentage of TUNEL positive cells among the total number of nuclei of the hippocampal region.

\section{Protein extraction and Western blot Analysis}

The samples were homogenized in RIPA buffer (Beyotime, China) with protease inhibitors ( $1 \mathrm{mM}$ phenylmethanesulfonylfluoride) on ice. The homogenates were then centrifuged at $12,000 \mathrm{~g}$ at $4^{\circ} \mathrm{C}$ for 20 min to remove tissue debris. The supernatant, stored at $-80^{\circ} \mathrm{C}$ \and subsequently used to Western blot. Prepare samples in EP tubes, add 4X SDS sample buffer so the total protein amount was $50 \mu \mathrm{g}$ per sample according to the protein amount measured by BCA protein assay (Beyotime, China), and then heated to $100^{\circ} \mathrm{C}$ for 5 minutes. Load samples and protein markers onto the $10 \%$ acrylamide gels (Beyotime, China). Set electrophoresis power pack to 80V (through the stacking gel), before increasing it to $120 \mathrm{~V}$ when the protein front reaches the separation gel. Then transferred to polyvinylidene difluoride membranes (PVDF, Millipore, USA) by electroelution at $4^{\circ} \mathrm{C}$. Membranes were blocked with $1 \mathrm{x}$ Tweencontaining Tris-buffered saline (TBST) containing 5\% nonfat dry milk with constant rocking for 2 hours at room temperature. The following primary antibody was incubated with the membrane under gentle agitation at $4^{\circ} \mathrm{C}$ overnight: anti-caspase 3 at 1:1000 dilution (Cell Signaling Technology, USA), anti-BDNF at 1:1000 dilution (abcam, USA), anti-proBDNF at 1:1000 dilution (alomone labs, Israel), anti-ERK1/2 at 1:1000 dilution (Cell Signaling Technology, USA), anti-phospho-ERK1/2 at 1:1000 dilution (Cell Signaling Technology, USA), anti-JNK at 1:1000 dilution (R\&D, USA), anti-phospho-JNK at 1:1000 dilution (abcam, USA), and anti- $\beta$-actin at 1:5000 dilution (proteintech, China). After washing three times with $1 \times$ TBST for 10 minutes each, the membranes were incubated for 2 hours with suitable horseradish peroxidase- 
conjugated secondary antibody (1:5000, ZSGB-BIO, China) with constant rocking for 2 hours at room temperature. After washing three times with $1 \times$ TBST for 10 minutes each, the membranes were incubated with sensitive chemoluminescence (ECL) substrate (Thermo, USA). Then read the membrane using a chemiluminescence imaging system (Azure C300, USA), and quantitative analysis of protein bands was measured using Image J. The protein expression of phospho-ERK1/2 or phosphor-JNK was normalized to the total ERK $1 / 2$ or JNK respectively. The band signals of other proteins were normalized to those of $\beta$-actin from the same samples.

\section{Immunohistochemistry Staining}

The paraffin blocks were separated from tissue holders, $3.5 \mu \mathrm{m}$ sections were then cut, placed on glass slides. The paraffin sections were allowed to dry at $67^{\circ} \mathrm{C}$ for $24 \mathrm{~h}$, and then immersed slides in xylene for deparaffinization and rehydrated sections by sequentially incubating with $100 \%, 95 \%, 80 \%$ and $60 \%$ ethanol for 5 minutes each. Rinsed sections with PBS three times for 5 minutes each. Transferred slides to a microwave-proof container and covered with citrate buffer, and heated in the microwave on high power for 7 minutes. Afterward, the brain sections were blocked with $3 \%$ hydrogen peroxide (ZSGB-BIO, China) at room temperature for 30 minutes for endogenous peroxidase ablation, and then washing with PBS. Sections were then blocked with $10 \%$ normal goat serum (ZSGB-BIO, China) for 45 minutes at room temperature. Discarded the goat serum and dropped the anti-caspase 3 (1:1000, Cell Signaling

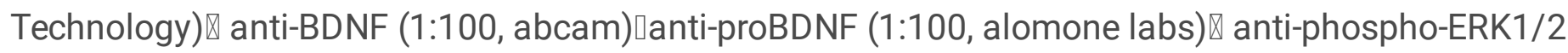
(1:100, abcam) and anti-phospho-JNK (1:100, abcam) primary antibody at $4^{\circ} \mathrm{C}$ overnight. Tissue sections were rinsed in PBS and dropped the goat anti-rabbit antibody (ZSGB-BIO, China) for 30 minutes at $37^{\circ} \mathrm{C}$, followed by incubation with the streptavidin-horseradish peroxidase (ZSGB-BIO, China) for 20 minutes at room temperature. Tissue sections were colored with diaminobenzidine (DAB, ZSGB-BIO, China) for 0.5-5 minutes and finished coloration with the distilled water, and then counterstained with hematoxylin. The sections were then dehydrated, cleared and mounted with neutral gums and covered with a coverslip. Negative control sections were carried out with the same steps as described above, but the primary antibody was replaced by PBS. Images were acquired at 400x using NIS-Elements F3.0 software linked to Nikon E800 microscope. Quantitative analysis of protein expression was performed using Image-Pro Plus. The quantitative analysis of target positive cells in the hippocampus was calculated by the optical density (OD) of positive cells by the area of that brain region.

\section{Statistical Analysis}

All statistical analyses were performed using SPSS version 21.0 software (IBM Corporation, Somers, NY, USA). All residuals followed normal distribution as detected by Shapiro-Wilk test. All values are presented as mean SD and analyzed using one-way analysis of variance (ANOVA), followed by LSD's posttest.

Significance was set at $\mathrm{P}<0.05$.

\section{Results}


All rats survived throughout the experiments. The percentage of TUNEL positive cells was increased in the isoflurane-treated rats compared with control rats $(P<0.001$, Fig. 1$)$. In addition, the expression of caspase 3 , which is a marker of apoptosis and cell death, detected by western blot increased in group Iso compared with control rats $(P<0.05$, Fig.2). Furthermore, the results of caspase 3 detected by immunohistochemistry staining were similar to those of the above $(P<0.05$, Fig. 3$)$.

\section{Neuroprotection induced by dexmedetomidine}

Dexmedetomidine attenuated apoptosis of neurons in the hippocampus induced by isoflurane. Additionally, compared with group Iso, in group Dex, the expression of caspase 3 detected by western blot was decreased $(P<0.001$, Fig.1). Likewise, the results of caspase 3 detected by immunohistochemistry significantly reduced by dexmedetomidine against isoflurane $(P<0.05$, Fig.3).

\section{The change of BDNF and proBDNF expression}

Western blot showed that the isoflurane reduced the expression of BDNF $(P<0.05$, Fig. 2$)$ which was significantly reversed by dexmedetomidine pretreatment $(P<0.05$, Fig.2). Furthermore, the protein expression of proBDNF in group Iso is significantly higher than group Con $(P<0.05, F i g .2)$, and dexmedetomidine pretreatment reversed the increase of proBDNF $(\mathrm{P}<0.05$, Fig.2). Immunohistochemical detection of BDNF and proBDNF had the same results consistent with the results of Western Blot $(P<0.05$, Fig.3).

\section{The expression of phospho-ERK and phospho-JNK}

The expression of phospho-ERK detected by western blot was reduced in group Iso( $P<0.05$, Fig.4), and this was significantly reversed by dexmedetomidine pretreatment $(P<0.05$, Fig.4). In addition, the rat's exposure to isoflurane increased the protein expression of phospho-JNK increased significantly compared with group Con $(\mathrm{P}<0.05$, Fig.4), and dexmedetomidine pretreatment reversed the change of phospho-JNK ( $\mathrm{P}<0.05$, Fig.4). Furthermore, compared with group Dex, the phosphorylation of ERK and JNK were significantly reduced by the inhibitors of TrkB and p75NTR $(P<0.05$, Fig.4). Meanwhile, immunohistochemical detection of phospho-ERK and phospho-JNK had the same results $(P<0.05$, Fig.5).

\section{Discussion}

The results of our study manifested that pretreatment with dexmedetomidine led to a significant decrease in neuroapoptosis in the hippocampus in neonatal rats in isoflurane anesthesia. These data demonstrate that pretreatment with dexmedetomidine may offer a strategy for neonatal brain protection during anesthesia of isoflurane. This protection was associated with activation of BDNF-mediated phospho-ERK, and a decrease of proBDNF-mediated phospho-JNK which are important for neuron survival. To our knowledge, this is the first time to demonstrate that the dexmedetomidine could attenuate isofluraneinduced neuroapoptosis, as evidenced by the decrease of the production of proBDNF and thereby inhibiting the activation of JNK. 
Consistent with other evidence that apoptotic neurodegeneration can be induced by exposure to drugs during the brain growth spurt, neonatal exposure to isoflurane was shown to induce widespread apoptosis in several major brain regions [1] [20]. The growth and development of the brain involve various processes, including neurogenesis, neural differentiation, migration, degeneration, apoptosis, and synapse formation [2], which are closely related to the aging process. In humans, the last trimester of pregnancy to the age of 2-3 years is the rapid development period of the brain, which is equivalent to rats from the late embryonic stage to 2-3 weeks after birth, and the brain synapse appeared rapidly during this period [21] [22]. Synapse is an important structure of information transmission among neurons. It has been hypothesized that the developing nervous system is more susceptible to certain neurotoxic injury than the mature brain because of the complexity and temporal features associated with brain development [1]. Anesthesia-induced neuroapoptosis is age-dependent, and the developing brain is most vulnerable at the age of 7 days [1] [23] [24].

Isoflurane is one of the most frequently used volatile anesthetics for induction and maintenance of anesthesia during surgery. Because isoflurane has rapid induction and recovery properties with limited irritation to the airway, it is particularly beneficial for infants undergoing the surgical process. Isoflurane exerts its effects through the GABA receptor and NMDA receptor. Exposure to isoflurane leads to neuronal apoptosis of immature brain in neonatal rats, which influence the function of learning and memory in adulthood [24]. The hippocampus is closely related to the function of learning and memory. It is reported that isoflurane induced neuronal apoptosis and damage in the cognitive function in adulthood. Its mechanism of toxicity comprises a wide range of factors, such as calcium concentration, mitochondrial pathway and inflammatory factor release [25] [26]. In the preliminary experiment, we found that 7-day-old rats exposed to $2 \%$ isoflurane, which is approximately equivalent to $0.8 \mathrm{MAC}$ [27], for 4 hours, induced significant apoptosis in hippocampal regions, and that is consistent with previous reports [28]. Some reports showed that neurotoxicity of isoflurane was associated with down-regulation of phosphor-ERK and up-regulation of phosopho-JNK [28]. Head et al. found that isoflurane neurotoxicity in the neonates is mediated by enhanced proBDNF/p75NTR and reduced BDNF [9].

The results of our study suggest that dexmedetomidine may be a clinical accessible tool to be used in children. a2-Adrenergic receptors are functionally coupled to $G$ proteins from early in development [29]. and have an inhibitory influence on neuronal activity [30]. As a newer a2-adrenergic agonist, dexmedetomidine is often used as a sedative and anesthetic adjuvant for patients because of its anesthetic-sparing effects and properties of anti-inflammation and cardiovascular protection [31] [32] and neuroprotection [12] [33].

Dexmedetomidine provided neuroprotection against isoflurane-induced neuroapoptosis in a dosedependent manner. Some reports [13] [34] found that dexmedetomidine lacks neurotoxicity even at extremely high dose such as $75 \mu \mathrm{g} / \mathrm{kg}$. Single administration of dexmedetomidine $75 \mu \mathrm{g} / \mathrm{kg}$ provides a similar effect to that of applying $25 \mu \mathrm{g} / \mathrm{kg}$ for three times during a 6 hours isoflurane exposure. However, Neuroprotective effects of dexmedetomidine had ceiling-effect, more than $12.5 \mu \mathrm{g} \square \mathrm{kg}-1 \mathrm{~h}$ - 1 can't produce a larger anti-apoptotic effect. Thus, in our study dexmedetomidine of $50 \mu \mathrm{g} / \mathrm{kg}$ induced an adequate 
effect of neuroprotection during 4 hours of exposure to isoflurane. We observed that pretreatment with dexmedetomidine at a dosage of $50 \mu \mathrm{g} / \mathrm{kg}$ significantly attenuated apoptosis in the hippocampal region of developing rats.

The neuroprotective effects of a2-adrenergic agonists have been studied for more than 20 years [35], however, the mechanisms of protection remain unknown. a2-Adrenoceptor is a subtype of norepinephrine receptors, which is widely distributed in the central nervous system and regulated the function of each system. Dahmani found that dexmedetomidine played a neuroprotective role in cerebral ischemiareperfusion injury in rats by activation of a2-adrenoceptor and inhibition of adenylate cyclase [36]. It is known that dexmedetomidine attenuated neurotoxicity induced by isoflurane through upregulation of extracellular signal-regulated kinase (ERK1/2) and B cell lymphoma/leukemia-2 (BCL-2) by the activation of a2-adrenoceptor [18] [37]. Previous reports suggest the antiapoptotic effect of dexmedetomidine on axoneuron is mediated by a2-adrenoceptor [12] [13]. In addition, some of the neuroprotective effects of dexmedetomidine are mediated via activation of ERK pathway [16] [38] [39]. On the other hand, one current study manifested that the neuroprotection of dexmedetomidine was mediated by BDNF [19] [40]. BDNF is one neurotrophin that can regulate the survival, development, and function of neurons and has been associated with inhibition of apoptosis [41] [16] [42]. The proBDNF is a precursor peptide encoded by the BDNF gene. Intracellular proBDNF can be cleaved by fusin or proprotein convertases to mature BDNF. Binding of BDNF to its high-affinity receptor TrkB activates Ras/ERK signal transduction pathway, which is important in the formation of synaptic plasticity and the function of learning and memory. While proBDNF activated the JNK pathway mediated its high-affinity receptor p75NTR [43] [44]. Our experiment demonstrated that dexmedetomidine pretreatment resulted in an increased expression of BDNF. Furthermore, dexmedetomidine administered in neonatal rats lead to a decrease expression of proBDNF, which was the first time to show further evidence of neuroprotection. The experimental results show that the inhibitors of TrkB and p75NTR reduced the expression of phospho-ERK and phospho-JNK, which suggested that at least a part of the activation of ERK and JNK was mediated by BDNF and proBDNF.

Clinically, there is no definite conclusion about the neurodegeneration by anesthetics of infants. According to the process of neural development, the 7-day old rat is equivalent to the age of 2-3 years in the human. However, if the neurodegeneration induced by anesthetics as a clinical problem in pediatric anesthesia, the application of a2-adrenergic agonist requires further study in newborns. So, this study was reasonable and meaningful and provided theoretical support for clinical application.

A limitation of our study is that it is not possible to determine if the beneficial effects of dexmedetomidine were due to attenuation of the up-regulation of BDNF, the down-regulation of proBDNF independently, or there is an interaction underlying the action mechanism. Nonetheless, dexmedetomidine pretreatment exhibited neuroprotection against isoflurane anesthesia.

\section{Conclusions}


In summary, our study results support our hypothesis that dexmedetomidine attenuated isofluraneinduced neurotoxicity in the hippocampus of neonatal rats through enhanced BDNF signaling via TrkB and reduced proBDNF signaling via p75NTR.

\section{Abbreviations}

BDNF

Brain-derived neurotrophic factor

p75NTR

p75 neurotrophin receptor

proBDNF

Proneurotrophin

TrkB

Tropomyosin receptor kinase B

ERK

Extracellular signal-regulated kinase

JNK

c-Jun N-terminal kinase

\section{Declarations}

\section{Ethics approval and consent to participate}

This study was approved by the Institutional Animal Care and Use Committee at Shengjing Hospital, China Medical University (Animal Welfare Assurance) and performed in accordance with the principles of Laboratory Care, supervised by a qualified veterinarian.

\section{Consent for publication}

Not applicable.

\section{Availability of data and materials}

All data generated or analysed during this study are included in this published article.

\section{Competing interests}

The authors declare that there are no conflicts of interest.

\section{Funding}

This work was supported by grants from the National Natural Science Foundation of China (81271370), the National Natural Science Foundation of China (81800763) and the Natural Science Foundation of 
Liaoning Province of China (20180551076).

\section{Authors' contributions}

Xudong Ding had the idea for the article and worked on the project throughout. Hui Zhang and Shiwei Sun are responsible for the animal model establishment. Morphological results of this paper was performed by Chenguang Ma. Ningning Zheng was responsible for the writing and English polishing of this paper. Hongtao Liu provided the overall theoretical guidance for the project.

\section{Acknowledgements}

The authors thank Yuxin Tong (Junior Faculty, Laboratory for Experimental Research, Shengjing Hospital Affliated to China Medical University, Shenyang, Liaoning, 110004, China) for technical support.

\section{References}

[1] V. Jevtovic-Todorovic, R.E. Hartman, Y. Izumi, N.D. Benshoff, K. Dikranian, C.F. Zorumski, J.W. Olney, D.F. Wozniak, Early exposure to common anesthetic agents causes widespread neurodegeneration in the developing rat brain and persistent learning deficits., J. Neurosci. 23 (2003) 876-882. https://doi.org/10.1523/JNEUROSCI.23-03-00876.2003.

[2] G. Stratmann, J.W. Sall, L.D. V May, J.S. Bell, K.R. Magnusson, V. Rau, K.H. Visrodia, R.S. Alvi, B. Ku, M.T. Lee, R. Dai, Isoflurane differentially affects neurogenesis and long-term neurocognitive function in 60-day-old and 7-day-old rats., Anesthesiology. 110 (2009) 834-848.

https://doi.org/10.1097/ALN.0b013e31819c463d.

[3] M. Satomoto, Y. Satoh, K. Terui, H. Miyao, K. Takishima, M. Ito, J. Imaki, Neonatal exposure to sevoflurane induces abnormal social behaviors and deficits in fear conditioning in mice., Anesthesiology. 110 (2009) 628-637. https://doi.org/10.1097/ALN.0b013e3181974fa2.

[4] H.H. Hansen, T. Briem, M. Dzietko, M. Sifringer, A. Voss, W. Rzeski, B. Zdzisinska, F. Thor, R. Heumann, A. Stepulak, P. Bittigau, C. Ikonomidou, Mechanisms leading to disseminated apoptosis following NMDA receptor blockade in the developing rat brain., Neurobiol. Dis. 16 (2004) 440-453. https://doi.org/10.1016/j.nbd.2004.03.013.

[5] D. Ma, P. Williamson, A. Januszewski, M.-C. Nogaro, M. Hossain, L.P. Ong, Y. Shu, N.P. Franks, M. Maze, Xenon mitigates isoflurane-induced neuronal apoptosis in the developing rodent brain., Anesthesiology. 106 (2007) 746-753. https://doi.org/10.1097/01.anes.0000264762.48920.80.

[6] L.X. Lu, J.-H. Yon, L.B. Carter, V. Jevtovic-Todorovic, General anesthesia activates BDNF-dependent neuroapoptosis in the developing rat brain., Apoptosis. 11 (2006) 1603-1615. https://doi.org/10.1007/s10495-006-8762-3. 
[7] H. Viberg, E. Pontén, P. Eriksson, T. Gordh, A. Fredriksson, Neonatal ketamine exposure results in changes in biochemical substrates of neuronal growth and synaptogenesis, and alters adult behavior irreversibly., Toxicology. 249 (2008) 153-159. https://doi.org/10.1016/j.tox.2008.04.019.

[8] B.P. Lemkuil, B.P. Head, M.L. Pearn, H.H. Patel, J.C. Drummond, P.M. Patel, Isoflurane neurotoxicity is mediated by p75NTR-RhoA activation and actin depolymerization., Anesthesiology. 114 (2011) 49-57. https://doi.org/10.1097/ALN.0b013e318201dcb3.

[9] B.P. Head, H.H. Patel, I.R. Niesman, J.C. Drummond, D.M. Roth, P.M. Patel, Inhibition of p75 neurotrophin receptor attenuates isoflurane-mediated neuronal apoptosis in the neonatal central nervous system., Anesthesiology. 110 (2009) 813-825. https://doi.org/10.1097/ALN.0b013e31819b602b.

[10] M.A.E. Ramsay, D.L. Luterman, Dexmedetomidine as a total intravenous anesthetic agent., Anesthesiology. 101 (2004) 787-790. https://doi.org/10.1097/00000542-200409000-00028.

[11] S.M. Walker, R.F. Howard, K.A. Keay, M. Fitzgerald, Developmental age influences the effect of epidural dexmedetomidine on inflammatory hyperalgesia in rat pups., Anesthesiology. 102 (2005) 12261234. https://doi.org/10.1097/00000542-200506000-00024.

[12] D. Ma, M. Hossain, N. Rajakumaraswamy, M. Arshad, R.D. Sanders, N.P. Franks, M. Maze, Dexmedetomidine produces its neuroprotective effect via the alpha 2A-adrenoceptor subtype., Eur. J. Pharmacol. 502 (2004) 87-97. https://doi.org/10.1016/j.ejphar.2004.08.044.

[13] R.D. Sanders, J. Xu, Y. Shu, A. Januszewski, S. Halder, A. Fidalgo, P. Sun, M. Hossain, D. Ma, M. Maze, Dexmedetomidine attenuates isoflurane-induced neurocognitive impairment in neonatal rats., Anesthesiology. 110 (2009) 1077-1085. https://doi.org/10.1097/ALN.0b013e31819daedd.

[14] D.S. Carollo, B.D. Nossaman, U. Ramadhyani, Dexmedetomidine: a review of clinical applications., Curr. Opin. Anaesthesiol. 21 (2008) 457-461. https://doi.org/10.1097/AC0.0b013e328305e3ef.

[15] M. Benggon, H. Chen, R. Applegate, R. Martin, J.H. Zhang, Effect of dexmedetomidine on brain edema and neurological outcomes in surgical brain injury in rats., Anesth. Analg. 115 (2012) 154-159. https://doi.org/10.1213/ANE.0b013e31824e2b86.

[16] Y.-M. Zhu, C.-C. Wang, L. Chen, L.-B. Qian, L.-L. Ma, J. Yu, M.-H. Zhu, C.-Y. Wen, L.-N. Yu, M. Yan, Both $\mathrm{PI} 3 \mathrm{~K} / \mathrm{Akt}$ and ERK1/2 pathways participate in the protection by dexmedetomidine against transient focal cerebral ischemia/reperfusion injury in rats., Brain Res. 1494 (2013) 1-8.

https://doi.org/10.1016/j.brainres.2012.11.047.

[17] F. Zhang, T. Ding, L. Yu, Y. Zhong, H. Dai, M. Yan, Dexmedetomidine protects against oxygen-glucose deprivation-induced injury through the 12 imidazoline receptor-PI3K/AKT pathway in rat C6 glioma cells., J. Pharm. Pharmacol. 64 (2012) 120-127. https://doi.org/10.1111/j.2042-7158.2011.01382.x. 
[18] S. Dahmani, D. Rouelle, P. Gressens, J. Mantz, Characterization of the postconditioning effect of dexmedetomidine in mouse organotypic hippocampal slice cultures exposed to oxygen and glucose deprivation., Anesthesiology. 112 (2010) 373-383. https://doi.org/10.1097/ALN.0b013e3181 ca6982.

[19] V. Degos, T. Le Charpentier, V. Chhor, O. Brissaud, S. Lebon, L. Schwendimann, N. Bednareck, S. Passemard, J. Mantz, P. Gressens, Neuroprotective effects of dexmedetomidine against glutamate agonist-induced neuronal cell death are related to increased astrocyte brain-derived neurotrophic factor expression., Anesthesiology. 118 (2013) 1123-1132. https://doi.org/10.1097/ALN.0b013e318286cf36.

[20] S.A. Johnson, C. Young, J.W. Olney, Isoflurane-induced neuroapoptosis in the developing brain of nonhypoglycemic mice., J. Neurosurg. Anesthesiol. 20 (2008) 21-28. https://doi.org/10.1097/ANA.0b013e3181271850.

[21] L. Slomianka, I. Amrein, I. Knuesel, J.C. Sørensen, D.P. Wolfer, Hippocampal pyramidal cells: the reemergence of cortical lamination., Brain Struct. Funct. 216 (2011) 301-317. https://doi.org/10.1007/s00429-011-0322-0.

[22] L.-H. Yao, C.-H. Li, W.-W. Yan, J.-N. Huang, W.-X. Liu, P. Xiao, Cordycepin decreases activity of hippocampal CA1 pyramidal neuron through membrane hyperpolarization., Neurosci. Lett. 503 (2011) 256-260. https://doi.org/10.1016/j.neulet.2011.08.048.

[23] J.-H. Yon, J. Daniel-Johnson, L.B. Carter, V. Jevtovic-Todorovic, Anesthesia induces neuronal cell death in the developing rat brain via the intrinsic and extrinsic apoptotic pathways., Neuroscience. 135 (2005) 815-827. https://doi.org/10.1016/j.neuroscience.2005.03.064.

[24] A.W. Loepke, S.G. Soriano, An assessment of the effects of general anesthetics on developing brain structure and neurocognitive function., Anesth. Analg. 106 (2008) 1681-1707. https://doi.org/10.1213/ane.0b013e318167ad77.

[25] X. Wu, Y. Lu, Y. Dong, G. Zhang, Y. Zhang, Z. Xu, D.J. Culley, G. Crosby, E.R. Marcantonio, R.E. Tanzi, Z. $X i e$, The inhalation anesthetic isoflurane increases levels of proinflammatory TNF-a, IL-6, and IL-1 $\beta$., Neurobiol. Aging. 33 (2012) 1364-1378. https://doi.org/10.1016/j.neurobiolaging.2010.11.002.

[26] G. Stratmann, J.W. Sall, J.S. Bell, R.S. Alvi, L. d V May, B. Ku, M. Dowlatshahi, R. Dai, P.E. Bickler, I. Russell, M.T. Lee, M.W. Hrubos, C. Chiu, Isoflurane does not affect brain cell death, hippocampal neurogenesis, or long-term neurocognitive outcome in aged rats., Anesthesiology. 112 (2010) 305-315. https://doi.org/10.1097/ALN.0b013e3181ca33a1.

[27] G. Orliaguet, B. Vivien, O. Langeron, B. Bouhemad, P. Coriat, B. Riou, Minimum alveolar concentration of volatile anesthetics in rats during postnatal maturation., Anesthesiology. 95 (2001) 734-739. https://doi.org/10.1097/00000542-200109000-00028. 
[28] Y. Li, F. Wang, C. Liu, M. Zeng, X. Han, T. Luo, W. Jiang, J. Xu, H. Wang, JNK pathway may be involved in isoflurane-induced apoptosis in the hippocampi of neonatal rats., Neurosci. Lett. 545 (2013) 17-22. https://doi.org/10.1016/j.neulet.2013.04.008.

[29] H.K. Happe, D.B. Bylund, L.C. Murrin, Alpha-2 adrenergic receptor functional coupling to $G$ proteins in rat brain during postnatal development., J. Pharmacol. Exp. Ther. 288 (1999) 1134-1142.

[30] M.J. Millan, Descending control of pain., Prog. Neurobiol. 66 (2002) 355-474. https://doi.org/10.1016/s0301-0082(02)00009-6.

[31] R.M. Venn, A. Bryant, G.M. Hall, R.M. Grounds, Effects of dexmedetomidine on adrenocortical function, and the cardiovascular, endocrine and inflammatory responses in post-operative patients needing sedation in the intensive care unit., Br. J. Anaesth. 86 (2001) 650-656. https://doi.org/10.1093/bja/86.5.650.

[32] D.N. Wijeysundera, J.S. Naik, W.S. Beattie, Alpha-2 adrenergic agonists to prevent perioperative cardiovascular complications: a meta-analysis., Am. J. Med. 114 (2003) 742-752. https://doi.org/10.1016/s0002-9343(03)00165-7.

[33] K. Sato, T. Kimura, T. Nishikawa, Y. Tobe, Y. Masaki, Neuroprotective effects of a combination of dexmedetomidine and hypothermia after incomplete cerebral ischemia in rats., Acta Anaesthesiol. Scand. 54 (2010) 377-382. https://doi.org/10.1111/j.1399-6576.2009.02139.x.

[34] Z. Liao, D. Cao, X. Han, C. Liu, J. Peng, Z. Zuo, F. Wang, Y. Li, Both JNK and P38 MAPK pathways participate in the protection by dexmedetomidine against isoflurane-induced neuroapoptosis in the hippocampus of neonatal rats., Brain Res. Bull. 107 (2014) 69-78.

https://doi.org/10.1016/j.brainresbull.2014.07.001.

[35] W.E. Hoffman, M.A. Cheng, C. Thomas, V.L. Baughman, R.F. Albrecht, Clonidine decreases plasma catecholamines and improves outcome from incomplete ischemia in the rat., Anesth. Analg. 73 (1991) 460-464. https://doi.org/10.1213/00000539-199110000-00016.

[36] S. Dahmani, D. Rouelle, P. Gressens, J. Mantz, Effects of dexmedetomidine on hippocampal focal adhesion kinase tyrosine phosphorylation in physiologic and ischemic conditions., Anesthesiology. 103 (2005) 969-977. https://doi.org/10.1097/00000542-200511000-00011.

[37] R.D. Sanders, P. Sun, S. Patel, M. Li, M. Maze, D. Ma, Dexmedetomidine provides cortical neuroprotection: impact on anaesthetic-induced neuroapoptosis in the rat developing brain., Acta Anaesthesiol. Scand. 54 (2010) 710-716. https://doi.org/10.1111/j.1399-6576.2009.02177.x.

[38] S. Dahmani, A. Paris, V. Jannier, L. Hein, D. Rouelle, J. Scholz, P. Gressens, J. Mantz, Dexmedetomidine increases hippocampal phosphorylated extracellular signal-regulated protein kinase 1 and 2 content by an alpha 2-adrenoceptor-independent mechanism: evidence for the involvement of 
imidazoline 11 receptors., Anesthesiology. 108 (2008) 457-466.

https://doi.org/10.1097/ALN.0b013e318164ca81.

[39] M. Schoeler, P.D. Loetscher, R. Rossaint, A. V Fahlenkamp, G. Eberhardt, S. Rex, J. Weis, M. Coburn, Dexmedetomidine is neuroprotective in an in vitro model for traumatic brain injury., BMC Neurol. 12 (2012) 20. https://doi.org/10.1186/1471-2377-12-20.

[40] D.M. Juric, D. Loncar, M. Carman-Krzan, Noradrenergic stimulation of BDNF synthesis in astrocytes: mediation via alpha1- and beta1/beta2-adrenergic receptors., Neurochem. Int. 52 (2008) 297-306. https://doi.org/10.1016/j.neuint.2007.06.035.

[41] S. Finkbeiner, CREB couples neurotrophin signals to survival messages., Neuron. 25 (2000) 11-14. https://doi.org/10.1016/s0896-6273(00)80866-1.

[42] Q. Shi, P. Zhang, J. Zhang, X. Chen, H. Lu, Y. Tian, T.L. Parker, Y. Liu, Adenovirus-mediated brainderived neurotrophic factor expression regulated by hypoxia response element protects brain from injury of transient middle cerebral artery occlusion in mice., Neurosci. Lett. 465 (2009) 220-225. https://doi.org/10.1016/j.neulet.2009.08.049.

[43] N. Caza, R. Taha, Y. Qi, G. Blaise, The effects of surgery and anesthesia on memory and cognition., Prog. Brain Res. 169 (2008) 409-422. https://doi.org/10.1016/S0079-6123(07)00026-X.

[44] J.P. Mathew, M. V Podgoreanu, H.P. Grocott, W.D. White, R.W. Morris, M. Stafford-Smith, G.B. Mackensen, C.S. Rinder, J.A. Blumenthal, D.A. Schwinn, M.F. Newman, Genetic variants in P-selectin and C-reactive protein influence susceptibility to cognitive decline after cardiac surgery., J. Am. Coll. Cardiol. 49 (2007) 1934-1942. https://doi.org/10.1016/j.jacc.2007.01.080.

\section{Figures}

a

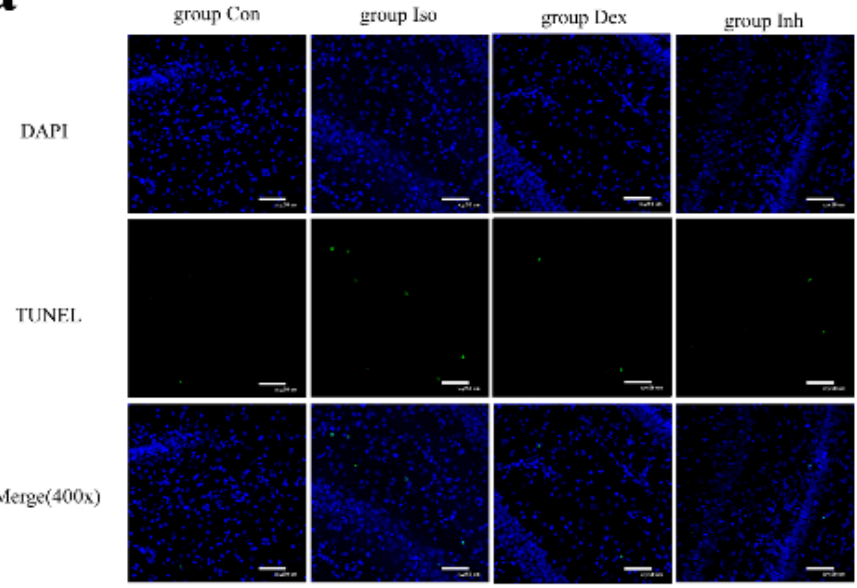

b

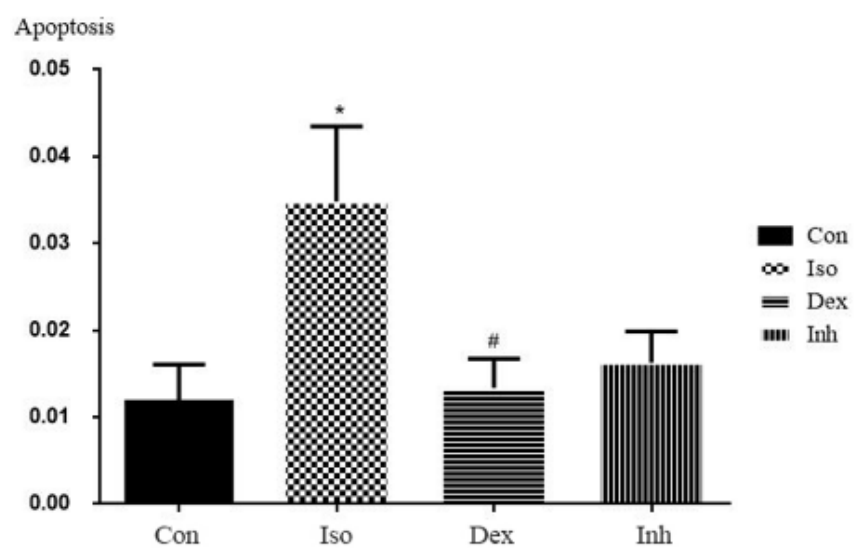


Figure 1

Dexmedetomidine attenuated the increase of isoflurane-induced neuroapoptosis in hippocampus of P7 rats $(n=10$ in each group, $P<0.05)$. Representative images of TUNEL in hippocampal region (a) Green staining indicated TUNEL-positive cells and blue staining indicated nuclei. Scan bar-50 $\mu \mathrm{m}$.

Quantification of TUNEL-positive in the hippocampal region by one-way ANOVA with LSD's multiple comparisons. Results are the means $\square S D(b) * P<0.05$ versus group Con; $\# P<0.05$ versus group Iso.

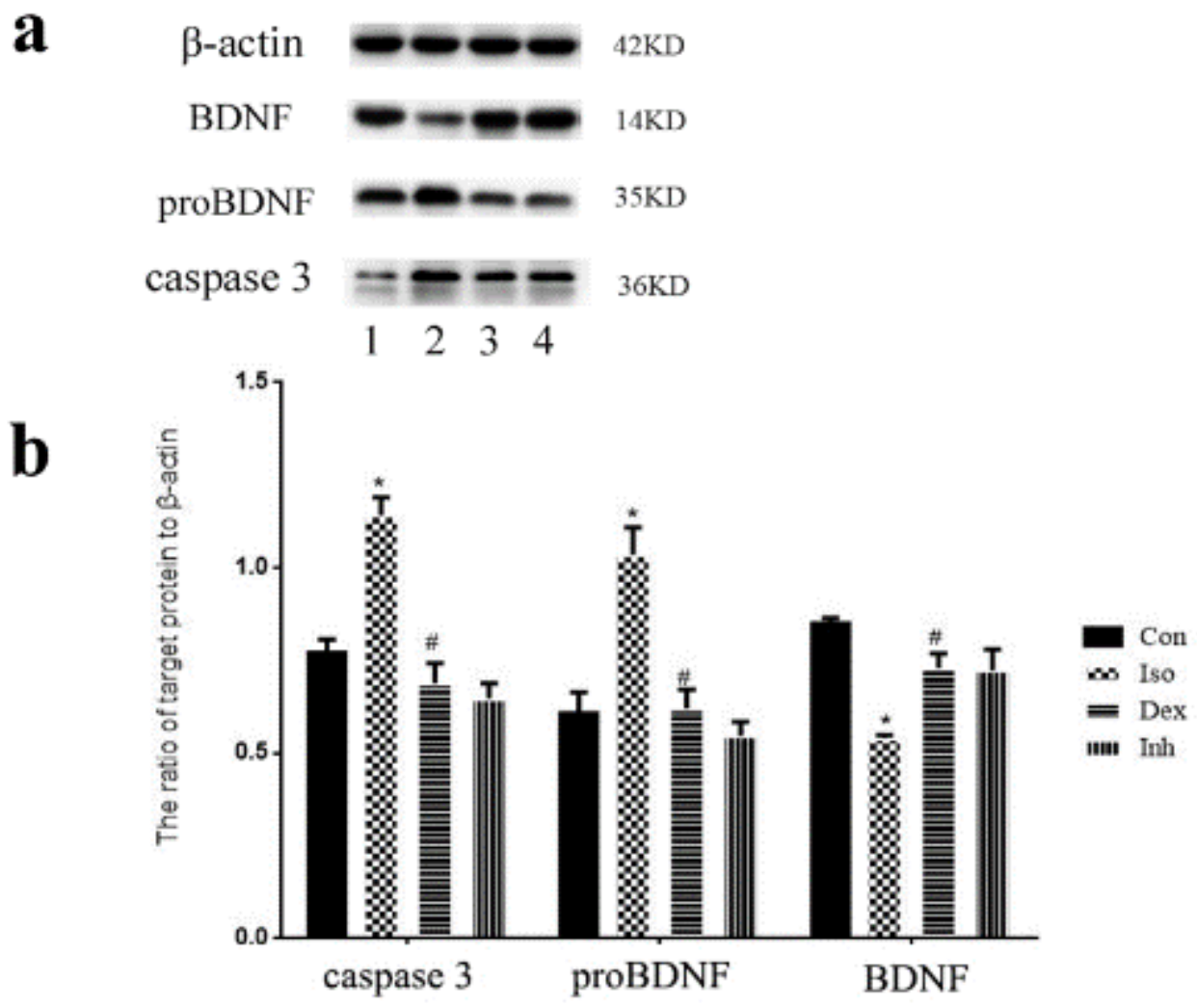

\section{Figure 2}

Dexmedetomidine reversed isoflurane-induced inhibition of BDNF and increase of proBDNF and caspase 3 in the hippocampus of $P 7$ rats $(n=8$ in each group $\triangle P<0.05)$. (a) Representative western blot of $B D N F$, proBDNF, caspase 3; (b) the quantitative analysis of BDNF, proBDNF, caspase 3 by one-way ANOVA with LSD's multiple comparisons. Results are the mean SD. Lane1:group Con; Lane2:group Iso; Lane3:group Dex; Lane4:group Inh. ${ }^{*} \mathrm{P}<0.05$ versus group Con; $\# \mathrm{P}<0.05$ versus group Iso. 

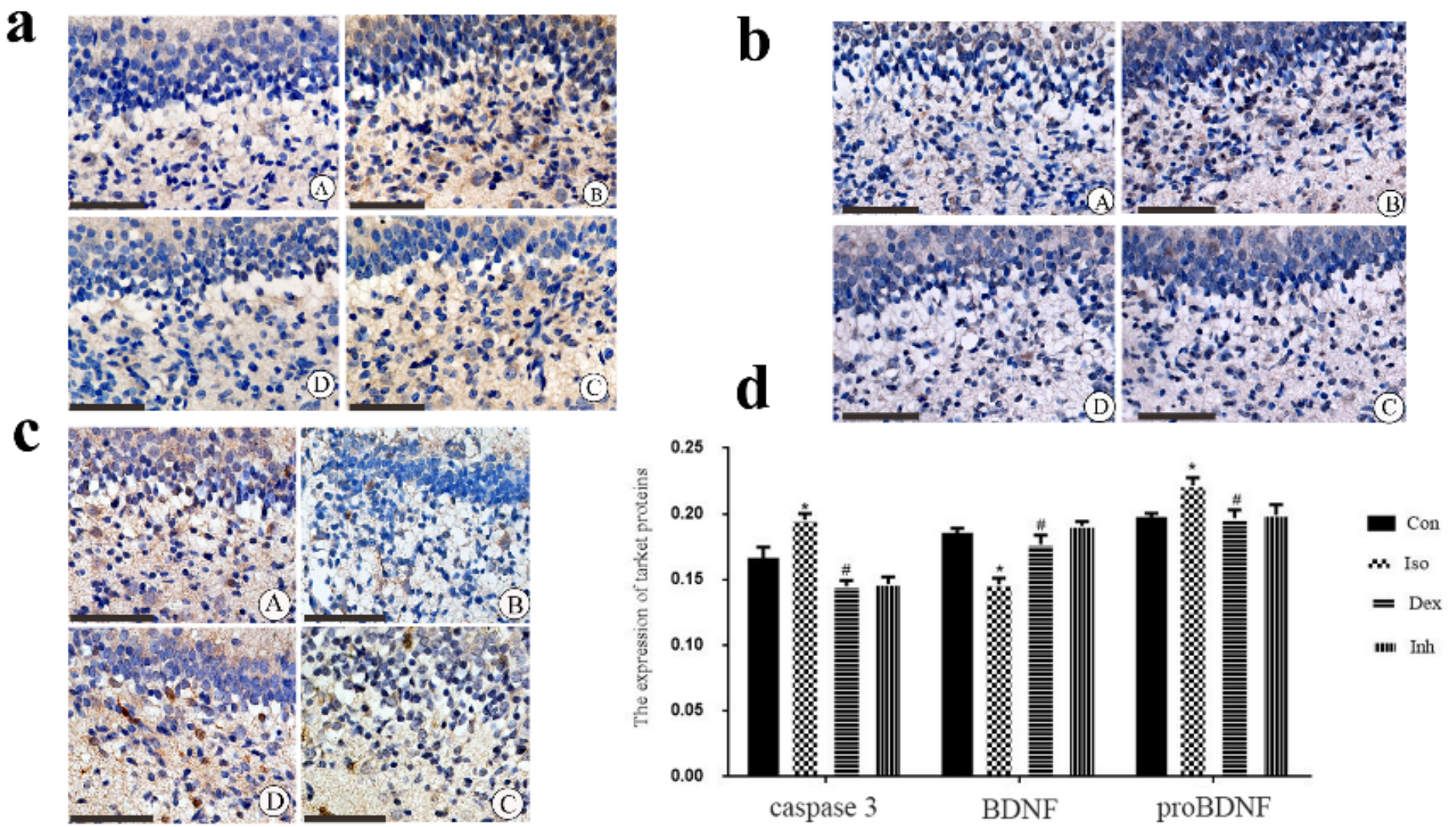

Figure 3

Dexmedetomidine reversed isoflurane-induced inhibition of BDNF and increase of proBDNF and caspase 3 in the hippocampus of $P 7$ rats $(n=10$ in each group $\mathbb{P}<0.05)$. (a)caspase 3; (b)proBDNF; (c)BDNF.

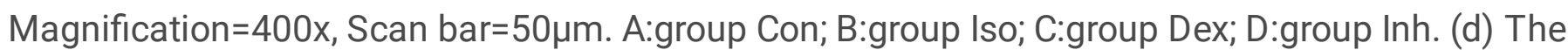
quantitative analysis of $B D N F$, proBDNF and caspase $3 .{ }^{*} P<0.05$ vs group Con; $\# P<0.05$ vs group Iso. 

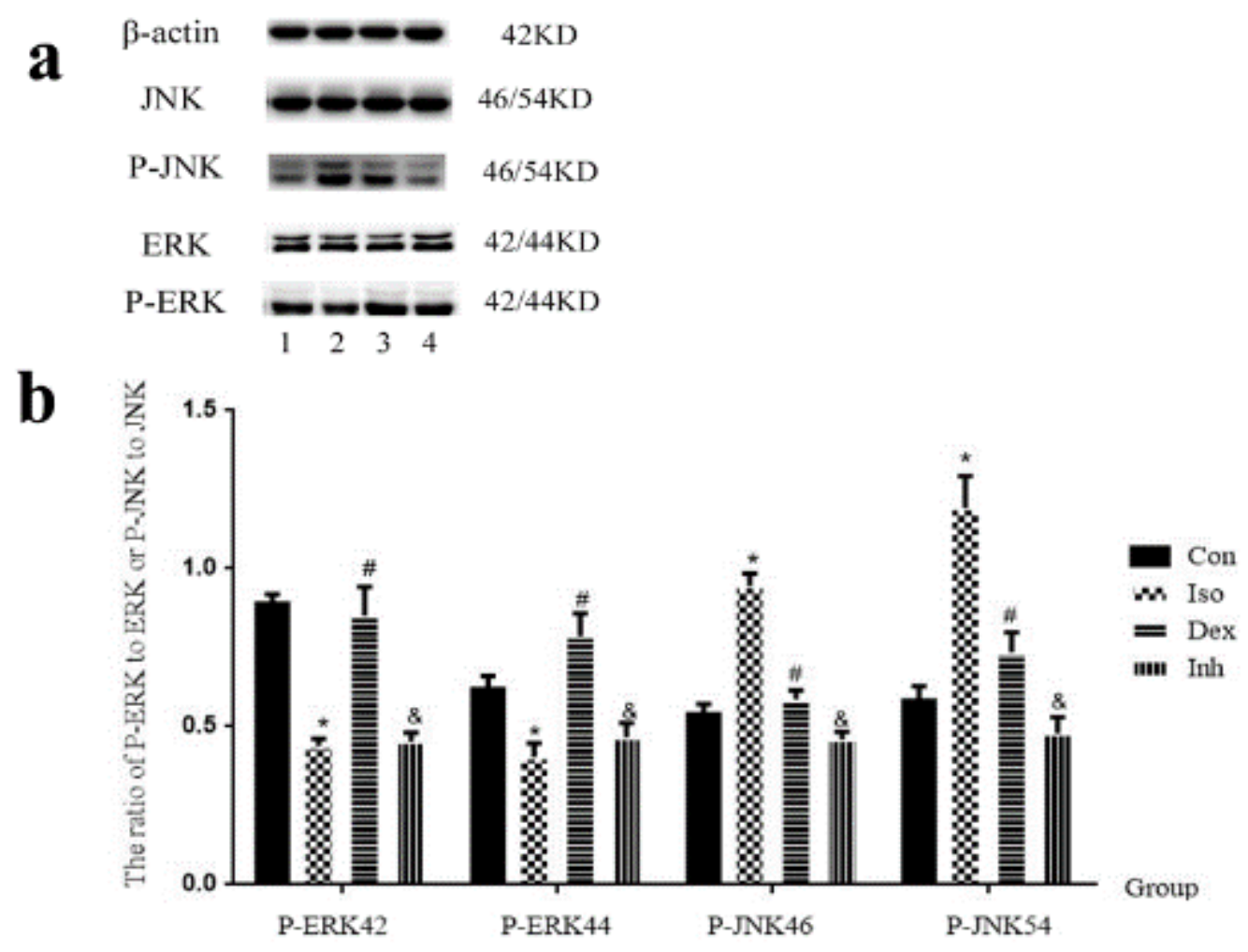

Figure 4

Dexmedetomidine reversed isoflurane-induced inhibition of P-ERK, and increase of P-JNK in the hippocampus of $\mathrm{P} 7$ rats $(\mathrm{n}=8$ in each group, $\mathrm{P}<0.05)$. (a)Representative western blot of P-ERK and P-JNK; (b) The histogram represented the quantitative analysis of P-ERK and P-JNK by one-way ANOVA with LSD's multiple comparisons. Results are the mean $\square S D$. Lane1:group Con; Lane2:group Iso; Lane3:group Dex; Lane4:group Inh. * $\mathrm{P}<0.05$ versus group Con; $\# \mathrm{P}<0.05$ versus group Iso; \& $\mathrm{P}<0.05$ versus group Dex. 

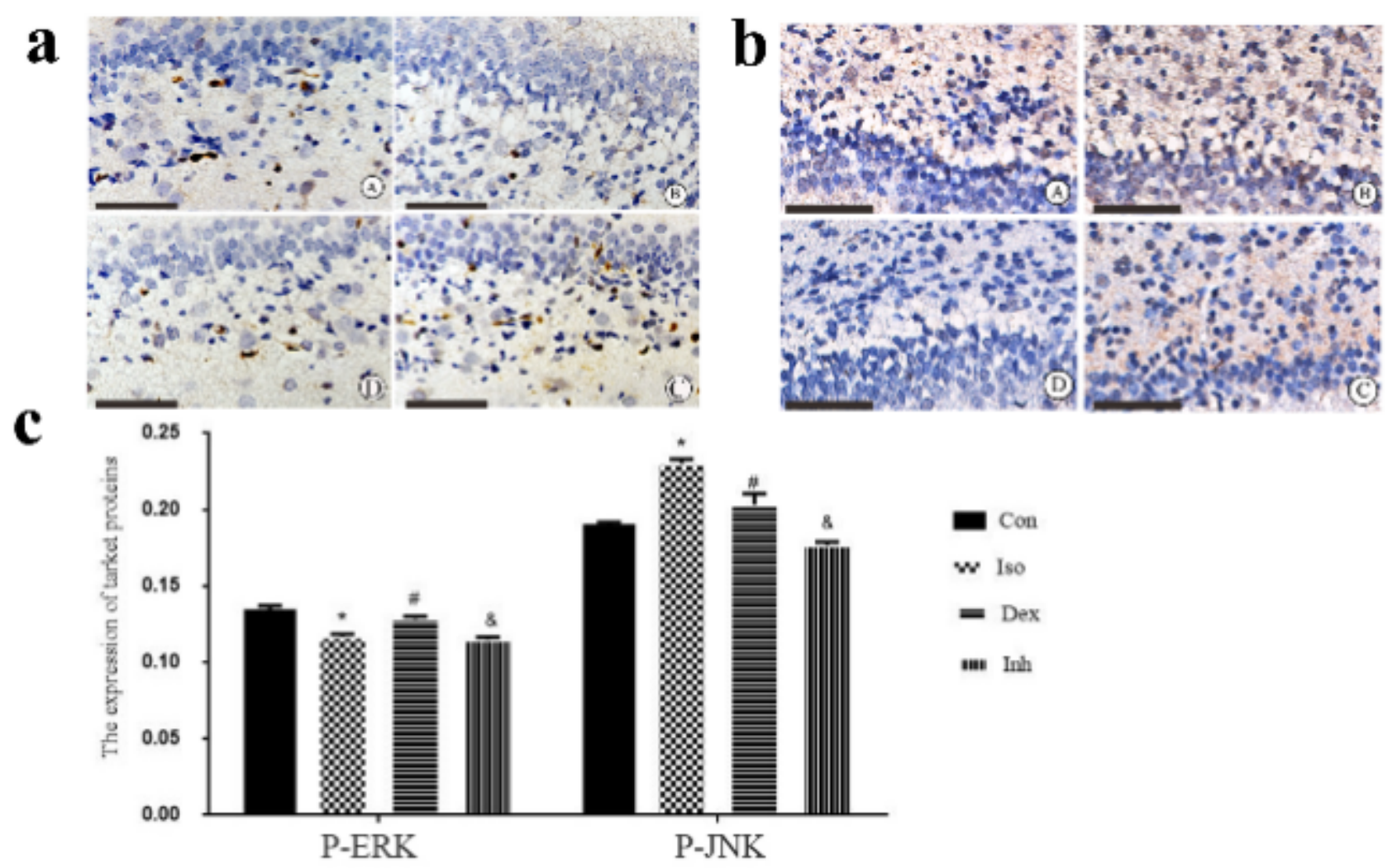

\section{Figure 5}

Dexmedetomidine reversed isoflurane-induced inhibition of P-ERK, and increase of P-JNK in the hippocampus of $P 7$ rats $(n=10$ in each group, $P<0.05)$. (b)(a),(b)Representative images of immunohistochemical $(\mathrm{IHC})$ staining in the hippocampal region from various experimental groups and the sample sections were illustrated with high magnification (400x). Scan bar=50 $\mu \mathrm{m}$. a:P-ERK; b:P-JNK. A:group Con; B:group Iso; C:group Dex; D:group Inh. (c) The quantitative analysis of P-ERK and P-JNK by one-way ANOVA with LSD's multiple comparisons. Results are the mean $\square S D$. ${ }^{*} P<0.05$ versus group Con; $\# \mathrm{P}<0.05$ versus group Iso; $\& \mathrm{P}<0.05$ versus group Dex. 